\title{
Etude De L'efficacité De L'huile De Thevetia Neriifolia Pour Le Contrôle De Anopheles Gambiae S.L Résistant Aux Pyréthrinoïdes
}

\begin{abstract}
Armand A. Akpo
Faculté des Sciences et Techniques, Université d'Abomey-Calavi, Bénin

\section{Daniel C. Chougourou}

Ecole polytechnique d'Abomey-Calavi, Département Génie de

l'Environnement, Université d'Abomey-Calavi, Bénin

\section{Razaki A. Osse}

Ecole de Gestion et d'Exploitation des Systèmes d'Elevage, Université

Nationale d'Agriculture, Bénin

\section{Joseph Dossou}

Département de Nutrition et Sciences Alimentaires, Faculté des Sciences

Agronomiques (FSA), Université d'Abomey-Calavi, Bénin
\end{abstract}

\section{Bruno Akinro}

Centre de Recherche Entomologique de Cotonou, Bénin

\section{Martin Akogbéto}

Faculté des Sciences et Techniques, Université d'Abomey-Calavi, Bénin

doi: 10.19044/esj.2017.v13n24p231 URL:http://dx.doi.org/10.19044/esj.2017.v13n24p231

\begin{abstract}
Effective control of pyrethroid-resistant malaria vectors requires new alternative measures. The purpose of this study is to test the effectiveness of Thevetia oil extracted from seed kernels to control malaria transmission by An. gambiae s.l. Sensitivity tests were carried out on larvae of Stages 3 and 4 of An. gambiae s.s of wild-type and pyrethroid-resistant Kis-kdr. The susceptible reference strain "Kisumu" served as a control. Mortality was read 24 hours and 48 hours after exposure. LD50 and 90 for 24 hours and 48 hours were determined using the log-probit method of determining the dose corresponding to a proportion. The high LD50s determined in 24 hours and 48 hours correspond to doses that kill $50 \%$ of Stage 3 larvae in wild populations in 24 hours and 48 hours. Low LD50s refer to stage 4 "Kisumu" larvae. The strong DL90 in 24 hours and 48 hours correspond respectively to stage 3 larvae of the Kis-kdr and wild-type strains. The comparison of the LD between the different strains shows the influence of the resistance on the sensitivity of the larvae of the strains resistant to the oil tested. However, the
\end{abstract}


larvicidal effect of Thevetia oil on the larvae of resistant strains may elicit formulations for alternative measures in vector resistance management to pyrethroids.

Keywords: Larvicidal activity, susceptibility test, An. gambiae s.s resistant, Anti - vector control

\section{Résumé}

Pour lutter efficacement contre les vecteurs du paludisme résistants aux pyréthrinoïdes, il est nécessaire de disposer des mesures alternatives nouvelles. Le but de cette étude est de tester l'efficacité de l'huile de Thevetia extraite des amandes des graines pour contrôler la transmission du paludisme assurée par An. gambiae s.l. Des tests de sensibilité ont été réalisés sur les larves des stades 3 et 4 de An. gambiae s.s des souches sauvage et Kis-kdr résistant aux pyréthrinoïdes. La souche sensible de référence «Kisumu» a servi de témoin. Les mortalités ont été lues 24 heures et 48 heures après exposition. Les DL 50 et 90 pour 24 heures et 48 heures ont été déterminées suivant la méthode log-probit de détermination de la dose correspondante à une proportion. Les fortes DL50 déterminée en 24 heures et 48 heures correspondent aux doses qui tuent $50 \%$ des larves de stade 3 des populations sauvages en 24 heures et 48 heures. Les faibles DL50 se rapportent aux larves de stade 4 «Kisumu». Les fortes DL90 en 24 heures et 48 heures correspondent respectivement aux larves de stade 3 des souches Kis-kdr et sauvage. La comparaison des DL entre les différentes souches montre l'influence de la résistance sur la sensibilité des larves des souches résistantes vis-à-vis de l'huile testée. Toutefois l'effet larvicide de l'huile de Thevetia sur les larves des souches résistantes pourra susciter des formulations pour des mesures alternatives dans la gestion de la résistance des vecteurs aux pyréthrinoïdes.

Mots clés: Activité larvicide, Test de sensibilité, An. gambiae s.s résistant, Lutte anti vectorielle

\section{Introduction}

L'espoir d'éradication du paludisme suscité par l'avènement des insecticides chimiques s'est éteint après un remarquable impact positif sur la réduction de la transmission du parasite et la nuisance des moustiques (Magesa et al., 1991; Beach et al., 1993; Akogbéto, 1995), laissant place à une nouvelle colonisation des écosystèmes par des vecteurs disposant de grande capacité de tolérance aux insecticides recommandés par l'OMS (Greorghiou et al., 1975; Sinegre et al., 1977; David et al., 2005; Müller et al., 2007; Corbel et al., 2007; Yadouléton et al., 2010). Dans le but de disposer des méthodes nouvelles, efficaces et diversifiées pour assurer une 
meilleure intervention pour la gestion de la résistance des vecteurs et la transmission du paludisme, des tests larvaires ont été conduits au laboratoire en vue d'évaluer l'efficacité de l'huile extraite des amandes de Thevetia neriifolia sur les larves des populations d'anophèles résistants. C'est une plante à intérêt médical à cause de sa propriété toxique (Garima et Amla, 2011; Neelam et Anil, 2014). L'activité insecticide des extraits a été prouvée par plusieurs dans le domaine agricole. En effet, les extraits des organes de la plante ont montré une activité biocide sur $C$. maculatus, une espèce de coléoptère ravageur des cultures (Mollah et Islam, 2007) et contre les charançons adultes de maïs (Wanyika et al., 2009). Cette plante dispose aussi d'une forte activité anti-termite et inhibitrice contre la croissance des souches de bactérie (Kareru et al, 2010), et contre la multiplication des virus à ARN (Garima et Amla, 2011). Les travaux réalisés par Chougourou et al., (2012) ont prouvé l'efficacité biocide de L'huile sur les larves de Musca domestica, un insecte constituant un intérêt majeur en santé humaine et dans le domaine agricole (Greenberg, 1971; Monzon et al., 1991; Fotedar et al., 1992; Blanchot, 1992; Sulaiman et al., 2000; Maldonado et al., 2003; Sukontason et al., 2007; Siriwattanarungsee, 2008).

La présente étude est consacrée à l'orientation de l'activité biocide connue de la plante à travers la mise en évidence de l'activité larvicide de l'huile extraite des graines pour la lutte contre An. gambiae s.s résistant aux pyréthrinoïdes de synthèse.

\section{Matériel et méthodes}

\section{Zone d'étude}

L'étude a été menée dans la ville de Cotonou, département du Littoral (Bénin) précisément dans les localités Zogbo, Ladji, Fifadji, Gbèdjromèdé, Houéyiho 1 et 2, Minontchou, Yénawa, Sourou-Léré. La ville de Cotonou a des caractéristiques géographiques qui sont favorables à la prolifération des moustiques. Elle présente une plaine sableuse sans relief, avec une nappe phréatique peu profonde et affleurant le sol (Akogbéto, 1992). Cette absence de relief, associée à un mauvais drainage des eaux, provoque des inondations pendant les saisons pluvieuses et favorise la multiplication des gîtes des Culicidae dont An.gambiae (Akogbéto et Yakoubou, 1999; Akogbéto, 1992). Par ailleurs, la ville de Cotonou est marquée par l'aménagement des terres et périmètres maraîchers, qui créent de véritables gîtes de Culicidae dont An.gambiae principal vecteur du paludisme (Akogbéto, 1992; Akogbéto, 1995). Elle abrite aussi le plus grand port du pays qui importe et exporte des conteneurs dans lesquels peuvent se cacher des moustiques venant d'autres horizons. Ces caractéristiques de la ville créent à ses habitants une nuisance culicidienne très forte. C'est l'une des villes les plus affectées par le paludisme au Bénin (MS, 2013). Les sites d'étude ont été choisis à cause de 
la fréquence et la permanence des gîtes larvaires qui occasionnent l'abondance et l'agressivité des moustiques. En effet, on y rencontre des flaques d'eau, des marécages temporaires et permanents, des pneus abandonnés ou stockés dans des garages à ciel ouverts...etc. Tous ces réservoirs constituent donc des gîtes potentiels favorables à la prolifération des moustiques.

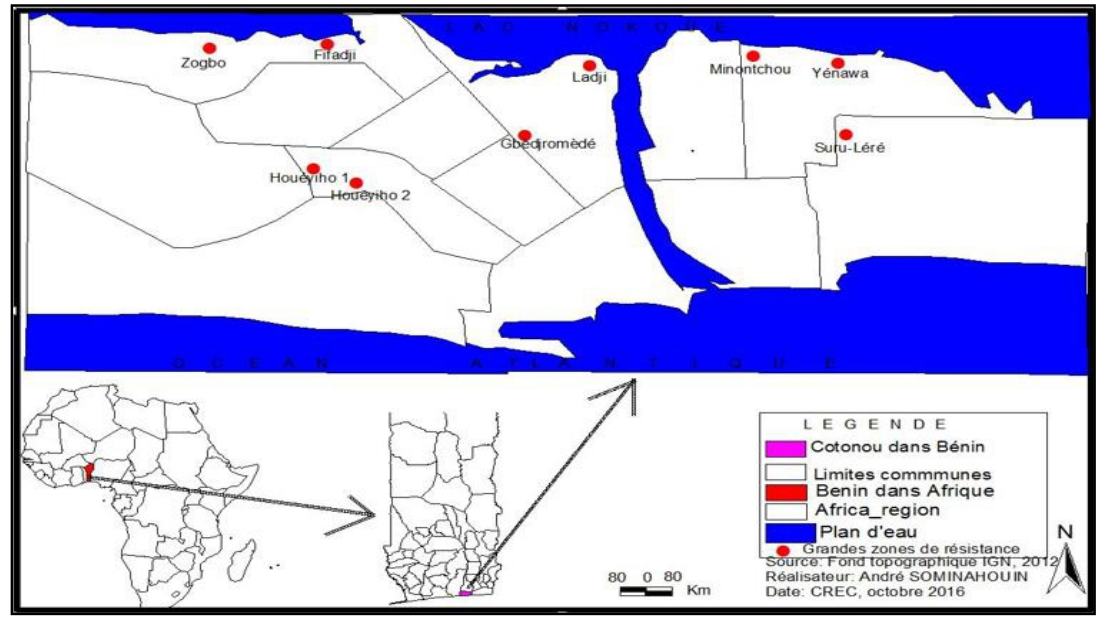

Figure 1: Situation des zones d'étude

\section{Matériel biologique}

Matériel animal

Les tests ont été réalisés sur les larves de An.gambiae s.s. Trois souches ont été utilisées. Il s'agit de la souche sensible «Kisumu» de génotype SS, donc dépourvue de l'allèle de résistance, la souche Kis-kdr de génotype RR résistant aux pyréthrinoïdes et aux organochlorés puis les populations sauvages caractérisées par une fréquence élevée de l'allèle de résistance du gène $\mathrm{Kdr}(>80 \%)$. Les souches de An. gambiae «Kisumu» et Kis-kdr sont élevées à l'insectarium du CREC (Centre de Recherche Entomologique de Cotonou). L'insectarium est constitué de salles où sont conditionnés séparément les stades pré imaginaux et imaginaux des différentes souches. Pour une bonne multiplication des moustiques, des conditions de température et d'hygrométrie doivent être réunies. A cet effet, les adultes sont conditionnés à une température de $26^{\circ} \mathrm{C} \pm 2^{\circ} \mathrm{C}$ grâce à un climatiseur qui fonctionne en permanence. La ponte des moustiques est assurée par des repas sanguins. Pour cela, des femelles âgées de trois à cinq jours sont gorgées pendant une vingtaine de minutes grâce à des lapins mâles immobilisés sur des cages de moustiques par un dispositif élaboré en conséquence. Après le repas sanguin, les femelles gorgées sont continuellement nourries au jus de miel à $10 \%$ dans du coton déposé sur le 
tulle moustiquaire des cages. Cela sert de nourriture pour les femelles gravides. Les œufs des différentes souches sont récoltés quotidiennement en retirant des cages les pondoirs après les pontes. Ces pondoirs sont systématiquement renouvelés après chaque retrait. Les œufs recueillis sont mis en eau (l'eau de robinet) dans des bacs en vue de leur éclosion. La salle d'élevage des larves est conditionnée à une plage de températures de $29^{\circ} \mathrm{C}$ à $32^{\circ} \mathrm{C}$ favorables au développement des stades pré imaginaux des moustiques et ceci à l'aide d'un radiateur de chauffage qui diffuse des calories par rayonnement. Les larves obtenues après éclosion sont nourries de croquette de chat dès le premier stade. L'eau d'élevage est renouvelée après 72 heures afin de garantir une bonne croissance des larves. Cela a permis d'obtenir des larves de stades 1, 2, 3, 4 et les pupes des différentes souches nécessaires pour la réalisation des tests. Les larves des moustiques sauvages sont récoltées dans les gîtes des sites d'étude de Cotonou aux moyens de louches, filtrées à l'aide d'un filtre à mailles serrées pouvant retenir les larves de différents stades et rincées à l'eau de robinet dans un bocal puis acheminées vers le laboratoire des tests du CREC.

\section{Matériel végétal}

Les matières végétales utilisées sont prélevées entre novembre 2014 et décembre 2015. Les graines mûres de Thevetia et tombées d'elles-mêmes ont été récoltées régulièrement dans un champ à Gamba, un village de la commune de Dassa où cette plante délimite un grand domaine privé. L'huile des amandes des graines est extraite à l'éthylacétate par soxhlet.

\section{Description de la méthode d'extraction de l'huile de Thevetia}

L'huile végétale obtenue à partir des amandes de Thevetia préalablement déshydratées par séchage à l'ombre est extraite par soxhlet au Laboratoire d'Etude et de Recherche en Chimie Appliquée à l'Ecole Polytechnique de l'Université d'Abomey-Calavi. L'extraction par soxhlet est une méthode simple et convenable permettant de répéter infiniment le cycle d'extraction avec du solvant frais jusqu'à l'épuisement complet du soluté dans la matière première déshydratée. Le soxhlet est composé d'un corps en verre, dans lequel est placée une cartouche en papier-filtre épais (une matière pénétrable pour le solvant), d'un tube siphon qui permet l'évacuation de la solution vers le ballon, d'un tube de distillation et d'un réfrigérant à eau qui permet la condensation des vapeurs de solvant dans la cartouche. Dans le montage, l'extracteur est placé sur un ballon contenant le solvant d'extraction. Le ballon contenant une réserve de solvant est chauffé afin de pouvoir faire bouillir son contenu. La cartouche contenant le solide à extraire est insérée dans l'extracteur, au-dessus duquel est placé le réfrigérant. Le ballon étant chauffé, le liquide est porté à l'ébullition. Les vapeurs du solvant 
passent par le tube de distillation et rentrent dans le réfrigérant pour être liquéfiées. Ensuite, le condensat retombe dans le corps de l'extracteur sur la cartouche, faisant ainsi macérer le solide dans le solvant. Le solvant condensé s'accumule dans l'extracteur jusqu'au niveau du sommet du tubesiphon, suivi par le retour dans le ballon du liquide de l'extracteur accompagné de substances extraites. Ainsi le solvant dans le ballon s'enrichit progressivement en composants solubles. L'extraction continue jusqu'à l'épuisement de la matière solide chargée dans la cartouche. L'éthylacétate est utilisé pour l'extraction de l'huile de Thevetia. Ce solvant a été choisi sur la base des propriétés physicochimiques (Température d'ébullition basse, liposolubilité) et toxiques (santé humaine et environnement). Les propriétés physicochimiques sur la base desquelles ce solvant est choisi se présentent dans le tableau suivant :

Tableau 1: Quelques caractéristiques du solvant utilisé pour l'extraction de l'huile de

Thevetia

\begin{tabular}{|c|c|c|c|}
\hline Solvants & Formules chimiques & $\begin{array}{c}\text { Température } \\
\text { d'ébullition }\end{array}$ & Avantages \\
\hline Ethylacétate & $\begin{array}{c}\mathrm{CH} 3-\mathrm{C}(=\mathrm{O})-\mathrm{O}-\mathrm{CH} 2- \\
\mathrm{CH} 3\end{array}$ & $77^{\circ} \mathrm{C}$ & $\begin{array}{c}\text {-Volatile et inflammable } \\
\text {-Neurotoxicité légère }\end{array}$ \\
\hline
\end{tabular}

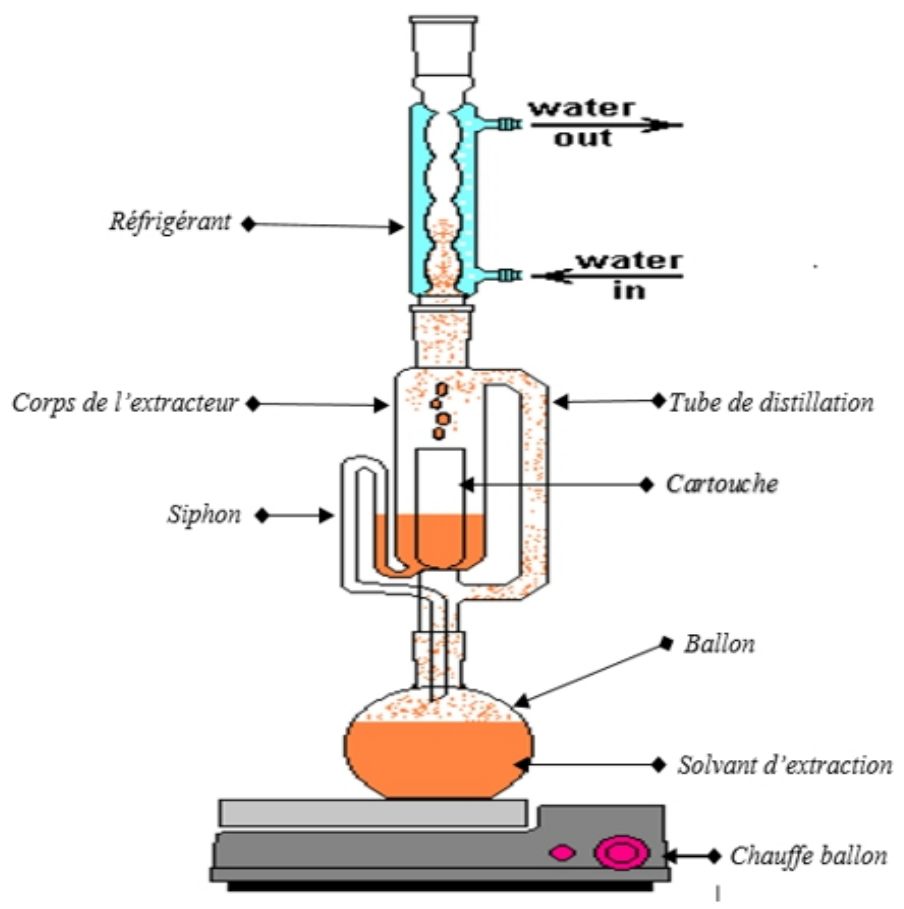

Figure 2: Schéma de 1'extracteur soxhlet 
La séparation du solvant de l'extrait est faite à l'aide de l'appareil appelé Rotavapor (voir figure). Dans cet appareil on réalise une évaporation sous vide en utilisant une pompe à vide avec une vanne de contrôle. Pendant l'évaporation le ballon est mis en rotation et plongé dans un bain liquide chauffé. L'appareil est muni d'un réfrigérant avec un ballon-collecteur de condensat. La rotation du ballon crée une surface d'échange plus grande et renouvelée permettant donc d'effectuer une évaporation rapide.

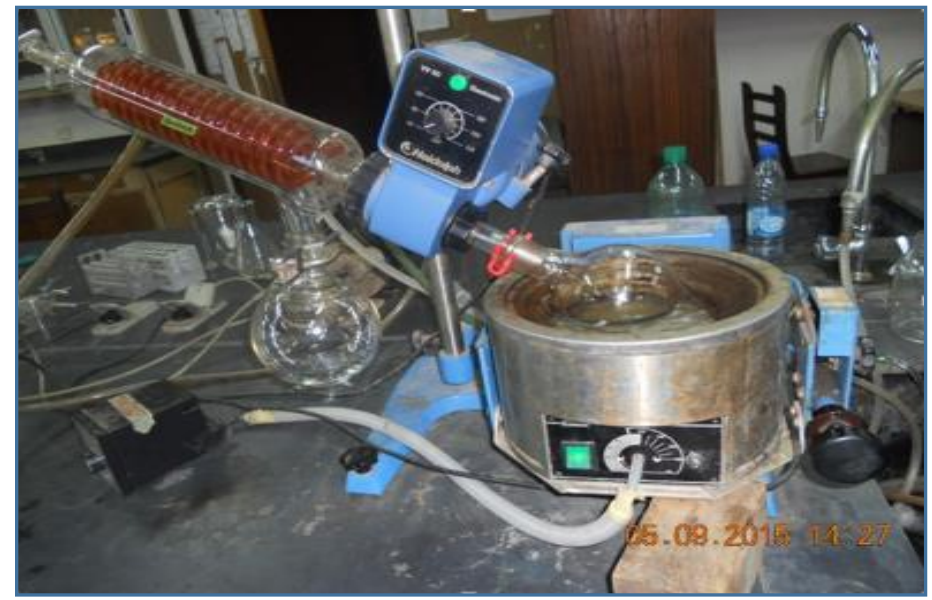

Photo 1: Photo de l'appareil rotavapor permettant la séparation des extraits du solvant.

Tests larvaire : Etude de l'efficacité de l'huile de Thevetia sur les larves de An. gambiae s.s résistants aux pyréthrinoïdes

Les tests de sensibilité ont été réalisés au CREC sur les larves des stades 3 et 4 des souches sensible «Kisumu», résistantes Kis-Kdr de laboratoire et sauvage conformément au protocole préconisé par l'Organisation Mondiale de la Sante (Abbott, 1925; OMS, 1963, 2004 \& 2014). Pour chaque stade larvaire des différentes populations de moustiques, des concentrations formulées à partir de l'huile de Thevetia ont été préparées plus les témoins avec cinq gobelets par concentration, contenant chacun 20 larves. Les concentrations sub-létales ont été choisies de manière à obtenir au moins un taux de mortalité de $100 \%$, à ce que certaines concentrations puissent donner un taux de mortalité comprise entre de 50 et $100 \%$ et allant de 5 à $50 \%$. Les larves mortes ont été dénombrées après 24 heures et 48 heures d'exposition. Les résultats sont exprimés en pourcentage de mortalité qui est déterminé en additionnant les nombres de larves mortes sur les réplications du test d'exposition par rapport au nombre total de larves de moustique exposées ainsi que dans les lots témoins selon la formule :

Mortalité au cours du test

$$
=\frac{\text { Nombre total de larves mortes }}{\text { Taille totale de l'échantillon }- \text { Nombre de pupes }} \times 100
$$


Lorsque le taux de mortalité enregistré au niveau des témoins est supérieur à $20 \%$, le test est invalide. Si la mortalité des témoins est supérieure à $5 \%$ et inférieure à $20 \%$ la mortalité observée est corrigée par la formule d'Abbott :

$$
\text { Mortalité corrigée }=\frac{\% \text { mortalité lors du test }-\% \text { mortalité témoin }}{100-\% \text { mortalité témoin }}
$$

Si la mortalité au niveau du témoin est inférieure à $5 \%$ elle peut être ignorée et bien, aucune correction n'est nécessaire.

Pour la détermination des doses létales des différents extraits sur les différents stades larvaires, le logiciel $\mathbf{R}$ Core Team (Version 3.3.1-2016) a été utilisé. La méthode utilisée est celle de log-probit de détermination de la dose correspondant à une proportion donnée.

\section{Détermination de la dose diagnostique pour la souche sauvage et les extraits des amandes de Thevetia.}

Les tests larvaires OMS permettent de diagnostiquer la sensibilité d'une population de vecteurs. Dans la présente étude, les tests sont réalisés dans le contexte de la gestion de la résistance de An. gambiae s.s, vecteur majeur du paludisme au Bénin (Akogbéto, 1992). Ils consistent à établir la dose diagnostique pour les souches de An. gambiae de référence «Kisumu» et sauvage et les différents extraits. Selon le comité OMS d'experts de la biologie des vecteurs et de la lutte antivectorielle, la dose diagnostique d'un insecticide est égale au double de la DL100 observée sur la souche sensible (OMS, 1992; Who, 2013). La DL100 correspond à la limite inférieure des doses qui tue $100 \%$ des populations cibles. Pour la présente étude, nous nous sommes basés sur les DL99 des stades 3 et 4 de la souche de référence «Kisumu» et des populations sauvages ont été établies. En effet les DL99 sont des doses qui tuent $99 \%$ des populations. Or les doses pouvant induire 99\% correspondent aux valeurs limites des intervalles de confiance des DL100. Par conséquent, les DL99 répondent à la logique des doses diagnostiques. Ensuite, les ratios des DL99 des stades respectifs de la souche sauvage sur celles de la souche sensible de référence ont été calculés. Puisque la dose diagnostique fait le double de la DL100 observée sur la souche sensible, un produit pourra susciter un espoir pour la lutte antivectorielle contre le paludisme lorsque le ratio $\mathrm{R}$ est inférieur ou égal à 2 . Lorsque $\mathrm{R}$ est supérieur à 2 , cela voudra indiquer un niveau élevé de la résistance vis-à-vis du produit testé.

\section{Formulation de l'huile de Thevetia}

L'huile de Thevetia est formulée à $1 \%$. Une solution mère de $1 \mathrm{mg} /$ $\mathrm{ml}$ dans l'éthanol a été préparée. A partir de cette solution mère, une gamme 
de dilutions a été effectuée en vue de varier les doses avec des pas de 0,01\%. La dispersion de l'huile dans l'eau et la stabilité de l'émulsion formée ont été réalisées après plusieurs essais de formulation au laboratoire d'étude et de Recherche en Chimie Appliquée à l'Ecole Polytechnique. $1 \mathrm{mg}$ d'huile nécessite $0,1 \mathrm{ml}$ d'éthanol et $0,1 \mathrm{mg}$ de lécithine pour stabiliser l'émulsion. Ainsi, pour préparer une solution mère de $200 \mathrm{ml}$ à $1 \mathrm{mg} / \mathrm{ml}, 200 \mathrm{mg}$ d'huile de Thevetia sont dissouts dans $20 \mathrm{ml}$ d'éthanol avec $20 \mathrm{mg}$ de lécithine. Le volume obtenu est complété d'eau distillée jusqu'à réaliser les $200 \mathrm{ml}$ de la solution mère.

\section{Impact à long terme d'une exposition des larves à la formulation de l'huile de Thevetia sur la biologie des survivants des larves exposées}

Les larves de stade 3 de la souche Kis-kdr sont exposées l'huile de Thevetia aux DL50 et DL90. Les larves ayant survécu sont récupérées, rincées à l'eau de robinet puis remises en observation dans les conditions d'élevage requises afin de suivre leur devenir après l'exposition à la matière larvicide. L'observation du comportement de ces larves ayant survécu a été faite sur une période de dix jours. Les pupes formées sont triées quotidiennement et mises en condition d'émergence. Les mortalités éventuelles des larves sont également dénombrées quotidiennement. L'eau d'élevage est renouvelée après 72 heures afin de garantir une bonne croissance des larves.

\section{Résultat}

\section{Tests d'efficacité de l'huile de Thevetia}

Les tests réalisés ont montré un effet biocide de l'huile de Thevetia sur les larves de An. gambiae s.s. Les mortalités enregistrées ont varié selon les doses et le temps d'exposition. Les résultats sont présentés dans le Tableau 2. Les doses de 0,$136 ; 0,11$ et $0,148 \mathrm{mg} / \mathrm{ml}$ ont induit en 24 heures $50 \%$ des larves de stade 3 respectivement des souches sensible de référence «Kisumu», kis-kdr et sauvage alors qu'il faut respectivement pour ces larves de $3^{\text {ème }}$ stade 0,$25 ; 0,3$ et $0,246 \mathrm{mg} / \mathrm{ml}$ pour induire $90 \%$. En 48 heures, les mortalités notées montrent qu'il faut moins de dose pour induire $50 \%$ et $90 \%$ des larves de stade 3 de ces différentes souches. Concernant les larves de stade 4 , les concentrations entrainant $50 \%$ de mortalité en 24 heures sont de 0,$048 ; 0,091$ et $0,144 \mathrm{mg} / \mathrm{ml}$ respectivement pour les souches de référence sensible «Kisumu», Kis-kdr et sauvage. En 48 heures, les résultats ont montré qu'il faut moins de dose pour entrainer les $50 \%$ de mortalité des larves du même stade. Les $90 \%$ de mortalité en 24 heures des larves de stade 4 des souches «Kisumu» de référence, Kis-kdr et sauvage sont obtenues avec les doses respectives de 0,$086 ; 0,2$ et $0,229 \mathrm{mg} / \mathrm{ml}$ alors qu'il faut 
respectivement pour ces trois souches 0,$064 ; 0,117$ et $0,156 \mathrm{mg} / \mathrm{ml}$ pour induire les $90 \%$ de mortalité en 48 heures.

Il ressort de ces résultats qu'il faut plus de dose en 24 heures d'exposition pour établir ma même efficacité de l'huile contre 48 heures d'exposition. Les larves de stade 4 des souches résistantes (Kis-kdr et sauvage) sont moins sensibles à l'huile de Thevetia par rapport à la souche sensible de référence «Kisumu». Il faut donc plus de dose pour tuer les larves de stade 4 des souches résistantes. Toutefois, les DL déterminées au stade 3 ne montrent pas l'influence de l'expression du gène $\mathrm{Kdr}$ sur la sensibilité des larves à 1'huile testée.

Tableau 2: Résultats des tests d'efficacité de l'huile de Thevetia. Les doses qui tuent $50 \%$ et $90 \%$ des larves des stades 3 et 4 des souches sensible de référence «Kisumu», Kis-Kdr (souche résistante de laboratoire) et des populations sauvages de Cotonou.

\begin{tabular}{|c|c|c|c|c|c|c|}
\hline & & & \multicolumn{2}{|c|}{24 heures } & \multicolumn{2}{|c|}{48 heures } \\
\hline & & & $\begin{array}{c}\text { Doses } \\
(\mathrm{mg} / \mathrm{ml})\end{array}$ & $I C(95 \%)$ & $\begin{array}{c}\text { Doses } \\
(\mathrm{mg} / \mathrm{ml})\end{array}$ & IC $(95 \%)$ \\
\hline \multirow{4}{*}{ Kisumu } & \multirow{2}{*}{$\mathbf{L 3}$} & DL50 & 0,136 & $\begin{array}{c}{[0,132-} \\
0,139]\end{array}$ & 0,087 & {$[0,083-0,090]$} \\
\hline & & DL90 & 0,25 & {$[0,24-0,26]$} & 0,2 & {$[0,19-0,21]$} \\
\hline & \multirow{2}{*}{ L4 } & DL50 & 0,048 & $\begin{array}{c}{[0,047-} \\
0,049]\end{array}$ & 0,032 & {$[0,030-0,033]$} \\
\hline & & DL90 & 0,086 & {$[0,082-0,09]$} & 0,064 & {$[0,061-0,067]$} \\
\hline \multirow{4}{*}{$K i s-k d r$} & \multirow{2}{*}{$\mathbf{L 3}$} & DL50 & 0,11 & {$[0,10-0,12]$} & 0,051 & {$[0,048-0,054]$} \\
\hline & & DL90 & 0,3 & {$[0,28-0,32]$} & 0,15 & {$[0,14-0,16]$} \\
\hline & \multirow{2}{*}{ L4 } & DL50 & 0,091 & $\begin{array}{c}{[0,088-} \\
0,095]\end{array}$ & 0,051 & {$[0,048-0,055]$} \\
\hline & & DL90 & 0,2 & {$[0,19-0,21]$} & 0,117 & {$[0,111-0,123]$} \\
\hline \multirow{4}{*}{ Sauvage } & \multirow{2}{*}{ L3 } & DL50 & 0,148 & $\begin{array}{c}{[0,144 \text { - }} \\
0,151]\end{array}$ & 0,108 & {$[0,105-0,111]$} \\
\hline & & DL90 & 0,246 & $\begin{array}{c}{[0,237-} \\
0,254]\end{array}$ & 0,204 & {$[0,196-0,212]$} \\
\hline & \multirow{2}{*}{ L4 } & DL50 & 0,144 & $\begin{array}{c}{[0,141-} \\
0,146]\end{array}$ & 0,073 & {$[0,070-0,076]$} \\
\hline & & DL90 & 0,229 & $\begin{array}{c}{[0,222 \text { - }} \\
0,236]\end{array}$ & 0,156 & {$[0,149-0,162]$} \\
\hline
\end{tabular}

DL50 et DL90 sont des concentrations qui tuent respectivement 50\% et 90\% des larves des stades 3 et 4 des souches sensible «Kisumu» de référence, Kis-kdr et sauvage en 24 heures et 48 heures post exposition à l'huile de Thevetia; IC (95\%) représente l'intervalle de confiance a $95 \%$. 
Détermination de Dose diagnostique de l'huile de Thevetia pour les larves de stade 3 et 4 des moustiques de la souche sauvage résistante et la souche sensible de référence «Kisumu».

Les résultats sont résumés dans le Tableau 3. L’analyse des résultats montre que les DL99 de l'huile sur les larves de stades 3 et 4 des populations sauvages sont supérieures à celles de la souche sensible de référence. Ce résultat indique une sensibilité faible des larves sauvages par rapport à la souche de référence. Les ratios DL99 sauvage/DL99Kisumu montrent qu'il faut moins du double de la dose qui tue $99 \%$ des larves de stade 3 de la souche de référence pour induire $99 \%$ de mortalité des larves de stade 3 de la souche sauvage. Au stade 4 la dose qui tue $99 \%$ des larves des populations sauvages est supérieure au double de la dose qui tue $99 \%$ des larves sensibles de référence «Kisumu». Il ressort de ces résultats que le portage de la résistance $\mathrm{kdr}$ n'influence pas considérablement l'efficacité de l'huile de Thevetia sur les larves de stade 3, ce qui n'est pas le cas au stade 4 bien que l'huile agit à forte dose sur les larves de stade 3 par rapport au stade 4 .

Tableau 3: Détermination des doses diagnostiques de l'huile de Thevetia pour les larves des stades 3 et 4 des populations de moustiques de souches sauvage et «Kisumu» de référence.

\begin{tabular}{|c|c|c|c|c|}
\hline & \multicolumn{2}{|c|}{ Sauvage } & \multicolumn{2}{|c|}{ Kisumu } \\
\hline & L3 & $\mathrm{L} 4$ & L3 & $\mathrm{L} 4$ \\
\hline DL99 & 0,415 & 0,334 & 0,373 & 0,139 \\
\hline IC-95\% & {$[0,388-0,444]$} & {$[0,317-0,351]$} & {$[0,352-0,394]$} & {$[0,130-0,148]$} \\
\hline \multicolumn{5}{|c|}{ Ratio DL99 Sauvage/DL99 Kisumu } \\
\hline & $\mathbf{L 3}$ & & 1,112 & \\
\hline & L4 & & 2,402 & \\
\hline
\end{tabular}

DL99 : Dose diagnostique entrainant 99\% de mortalité d'une population; Ratio DL99 Sauvage/DL99 sensible : rapport de dose diagnostique de souche sauvage sur celle de «Kisumu» de référence; IC (95\%) représente l'intervalle de confiance à $95 \%$.

\section{Espérance de vie des larves après 24 heures d'exposition à l'huile de Thevetia extrait à l'éthylacétate}

L'impact de l'huile de Thevetia sur la survie des larves après exposition à l'huile de Thevetia est évalué selon le protocole décrit dans le cas d'étude de l'efficacité du CNSL extrait à froid. Cette étude vise l'effet d'une exposition des larves à l'huile de Thevetia pendant 24 heures sur le développement des phases post exposition. L'étude a été réalisée sur les 
larves de stade 3 de An. gambiae de la souche Kis-kdr de génotype résistant $\mathrm{RR}$. Les résultats sont résumés dans le tableau $(\mathrm{x})$.

Sur 400 larves exposées à la DL50 en 24 heures, 214 ont survécu. Durant les 10 jours d'observation, seulement 11 larves ont évolué pour atteindre le stade de pupe. Les dernières pupes sont formées au cinquième jour d'observation alors que les dernières larves sont mortes au neuvième jour d'observation. Pour ce qui concerne les larves exposées à la DL90 en 24 heures, 14 seulement ont survécu sur 600 exposées. Une seule des survivants a évolué en pupe au cinquième jour d'observation et les dernières larves sont mortes au sixième jour d'observation. Contre ces deux faibles taux de formation des pupes sous l'effet des DL50 et DL90, 96\% de pupes sont obtenues dans le lot témoin.

Il ressort de ces résultats que l'huile de Thevetia tue les larves de $A n$. gambiae non seulement aux termes de 24 heures d'exposition mais retarde leur développement post-exposition, ce qui induit une mortalité tardive et massive des larves exposées. Une exposition à la DL90 réduit plus l'expérience de vie des larves comparativement à ce qui est enregistré à la DL50.

Tableau: Effet à long termes de l'huile de Thevetia extrait à l'éthylacétate sur les larves de stade 3 de An. gambiae Kis-Kdr aux DL50 et DL90

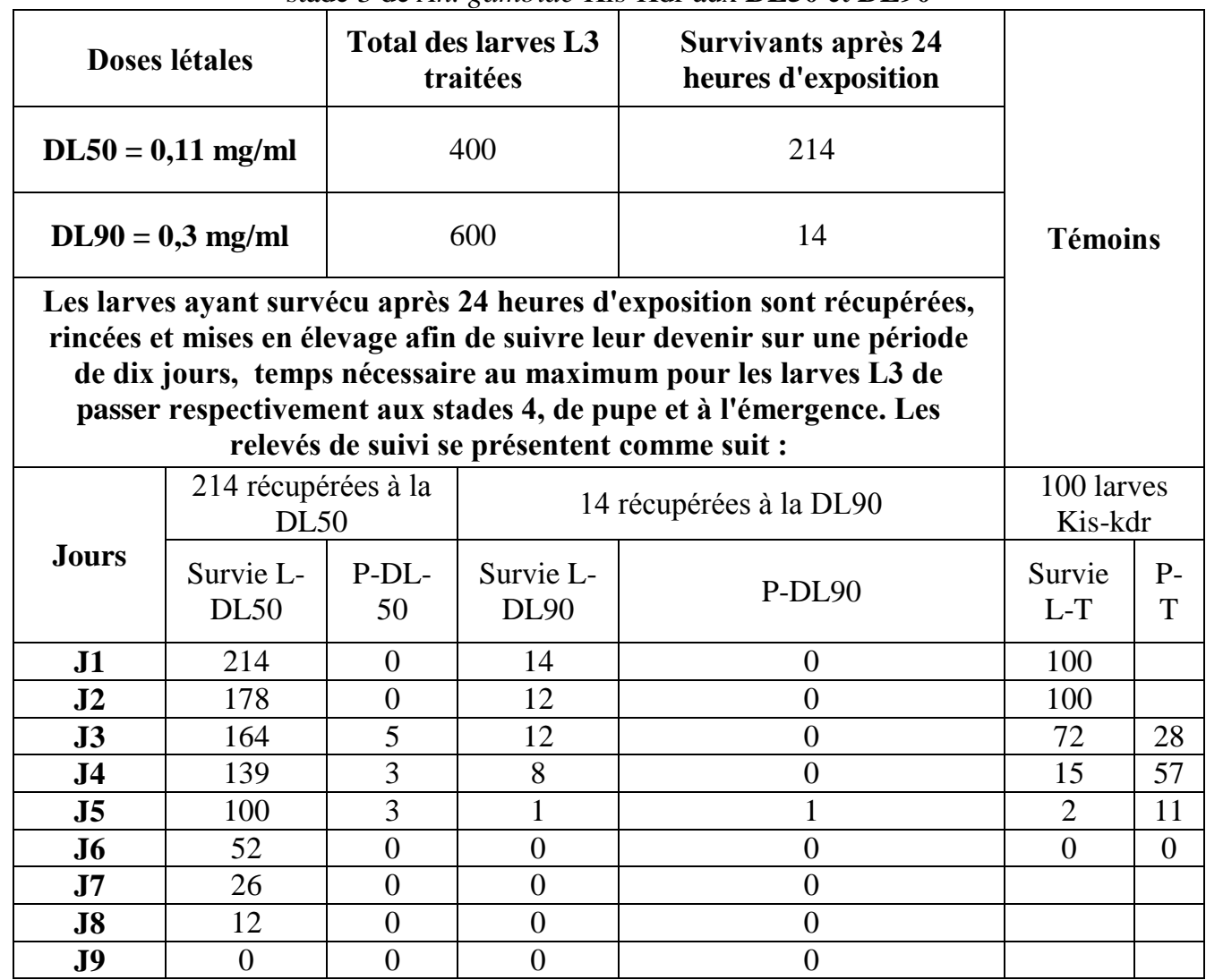




\begin{tabular}{|c|c|c|c|c|c|}
\hline J10 & 0 & 0 & 0 & 0 & \\
\hline $\begin{array}{l}\text { Pupes } \\
\text { (en \%) }\end{array}$ & \multicolumn{2}{|c|}{5,14} & \multicolumn{2}{|c|}{7,14} & 96 \\
\hline
\end{tabular}

DL50 et DL90 sont des doses létales qui tuent respectivement $50 \%$ et $90 \%$ des larves des stades 3 Kis-kdr; L3-DL50 et L3-DL90 sont les larves récupérées respectivement aux DL50 et DL90; P-DL50 et P-DL90 sont les pupes issues du développement des larves exposées respectivement aux DL50 et DL90 ; L3-T : larves de stade 3 témoins ; P-T3 : pupes issues du développement des larves témoins

\section{Discussion}

Dans le but d'étudier l'efficacité de l'huile de Thevetia pour une meilleure intervention dans la lutte contre les vecteurs du paludisme devenus résistants aux pyréthrinoïdes et assurer la préservation durable de l'environnement, des tests larvaires ont étés réalisés selon le protocole recommandé par l'Organisation mondiale de la santé (OMS, 1963; 2004 et 2014). Les larves des stades 3 et 4 de la souche sensible de référence «Kisumu» et des souches résistantes Kis-kdr de laboratoire et sauvage sont exposées à une gamme de concentrations de l'huile formulée de manière à induire des mortalités allant de 0 à $100 \%$. Les larves mortes sont dénombrées 24 heures et 48 heures après exposition aux différentes concentrations.

Les tests réalisés ont permis de déterminer les DL50 et DL90 de l'huile sur les larves des stades 3 et 4 de la souche sensible de référence «Kisumu» et des souches résistantes. Il ressort de l'analyse des résultats des tests réalisés que l'huile de Thevetia a une activité biocide sur les larves de An. gambiae des souches sensible et résistantes aux pyréthrinoïdes. Les doses létales pouvant tuer $50 \%$ et $90 \%$ des larves de stade 3 en 24 heures et 48 heures n'ont pas montré une sensibilité privilégiée remarquable des trois souches en fonction du portage du gène Kdr actif. Cette observation indique une bonne efficacité de l'huile de Thevetia pour la gestion de la résistance des vecteurs et les risques liés à l'usage des produits chimiques. Concernant les larves de stade 4 , les doses létales induisant $50 \%$ et $90 \%$ de mortalité en 24 heures et 48 heures montrent qu'il faut plus de dose de l'huile formulée tuant les larves de la souche sensible de référence «Kisumu» pour indure les mêmes mortalités des larves des souches résistantes. En effet, les larves de stade 4 des populations sauvages sont remarquablement moins sensibles à l'huile par rapport à celles des souches Kis-kdr qui sont aussi moins sensibles aux larves de stade 4 de la souche de référence «Kisumu». Toutefois, il apparait que l'huile de Thevetia présente plus d'efficacité sur les larves de stade 4 par rapport au stade 3. La vulnérabilité des larves de stade 4 serait liée à la profonde métamorphose qui caractérise ce stade conduisant à la formation des pupes. Plusieurs études ont montré que l'huile de Thevetia est essentiellement composé de thevetine A et B, de la thevotoxine, de la peruvoside, de ruvoside, de la neriifoline ...etc (Omolara et Samuel, 2007; 
Garima et Amla, 2011; Neelam et Anil, 2014). Ces composés seraient à l'origine de l'effet létal de l'huile. Les résultats obtenus à l'issu de nos travaux sont similaires à ceux de Brahim et al. (2006) qui ont montré l'intérêt des extraits des plantes dans la lutte contre les insectes. Il sera donc important d'élucider le mode d'action de l'huile de Thevetia à partir de la connaissance de la physiologie caractéristique des stades larvaires pour garantir la performance des formulations envisageables. La mise en évidence de l'effet larvicide de l'huile de Thevetia pourra contribuer à la mobilisation d'une bonne diversité de biopesticides extraits des plantes pour assurer les perspectives pour la lutte contre les vecteurs nuisibles en particuliers contre les moustiques vecteurs d'agents pathogènes (Ould El Hadj et al., 2006; Zouiten et al., 2006; Idrissi et Hermas, 2008; Kemassi et al., 2010 ; 2012 ;2014).

L'impact de l'huile sur les larves exposées a été étudié sur les larves de stade 3 de la souche Kis-kdr. Les larves exposées à la DL50 de l'huile formulée ont montré un retard de croissance caractérisé par un faible taux de formation de pupe, soit 5,14\% par rapport au témoin dont le taux de pupes formées est de $96 \%$. Ce ralentissement de croissance à la DL50 est noté également chez les larves exposées à la DL90 avec moins de formation de pupe suivie d'une mortalité progressive et massive des larves exposées à ces différentes doses. Les résultats des différents tests montrent que l'huile de Thevetia présente une activité biocide sur les larves des souches de $A n$. gambiae sensible et résistant aux pyréthrinoïdes de synthèse. La faible influence du gène $\mathrm{kdr}$ sur son efficacité pourra susciter son usage intégré dans la lutte contre les vecteurs du paludisme dans le contexte actuel de la gestion de la résistance des vecteurs et la protection de l'environnement.

Bien que les DL50 et DL90 de 1'huile aient montré la vulnérabilité des larves de stade 4 par rapport au stade 3 des différentes souches testées, les ratios des doses diagnostiques pour ces larves montrent une faible influence de la résistance sur la sensibilité des larves de stade 3 des populations sauvages. Le ratio des doses diagnostiques au stade 3 est de 1,112. Cette valeur montre clairement qu'il n'y a pas une différence très remarquable de sensibilité entre les larves de stade 3 de la souche sensible de référence et celle des populations sauvages. Le ratio des doses diagnostiques au stade 4 montrent l'impact de la résistance sur la sensibilité des larves de stade 4 des populations sauvages. La valeur enregistrée montre qu'il faut plus du double de la dose induisant $99 \%$ de mortalité des larves de la souche sensible pour obtenir la même efficacité sur les larves de terrain. La forte capacité des larves de stade 4 des moustiques sauvages à tolérer une forte dose d'huile de Thevetia justifie la forte adaptation aux polluants de nature et d'origine diverses qui sont au contact des laves dans leurs milieux de développement. Toutefois l'activité larvicide de l'huile serait un plus pour la 
mise en œuvre des moyens alternatifs de lutte contre les vecteurs résistant et pourrait être utilisée en synergie avec d'autres matières ayant des propriétés biocide dans des formulations pour la préservation des écosystèmes.

\section{Conclusion}

L'huile de Thevetia a une propriété biocide sur les larves de An. gambiae s.s de la souche sensible de référence «Kisumu» et de la souche sauvage. Les mortalités enregistrées sur la souche sauvage sont d'autant plus grandes que lorsque les doses appliquées sont fortes. La faible influence de la résistance sur la sensibilité des larves offre un bon moyen alternatif local pour le contrôle des vecteurs résistant aux produits chimiques.

\section{References:}

1. Abbott W.S., 1925 : "A method of computing the effectiveness of an insecticide", J. Econ. Entomo. vol. 18, pp. 265-267.

2. Akogbéto M. 1995 : Étude entomologique sur la transmission du paludisme côtièr lagunaire: cas d'un village construit sur un lac d'eau sâumatre. In: Annales de la Société Belge de Médecine Tropicale, pp. 219-227.

3. Akogbéto M., 1995 : Etude entomologique sur la transmission du paludisme côtier lagunaire: cas d'un village construit sur un lac d'eau saumâtre. Ann. Soc. Belge Med trop. 75, 219-227.

4. Akogbéto M., Chippaux J.-P. \& Coluzzi M., 1992 : Le paludisme urbain côtier à Cotonou (République du Bénin). Étude entomologique. Revue d'Epidémiologie et Santé Publique 40: 233239.

5. Beach R.F., Ruebush T.K., Sexton J.D., Bright P.L., Hightower A.W., Breman J.G., Mount D.L. \& Oloo A.J. 1993. Effectiveness of Permethrin-Impregnated Bed Nets and Curtains for Malaria Control in a Holoendemic Area of Western Kenya. The American Journal of Tropical Medicine and Hygiene 49: 290-300.

6. Blanchot P, 1992 : Nouveau répertoire bibliographique et nouvelles données biologiques sur les parasites de Musca domestica L. (Dipt.: Muscidae). École Pratique des Hautes Études, Biologie et Évolution des Insectes. 45 rue Buffon, F-75005. 42p.

7. Chougourou C. D., Dellouh P. L, Agbaka A., N'Guessan R. \& Gbénou J. D., 2012: Toxicité et effets répulsifs de certaines huiles extraites des plantes locales Béninoises sur la mouche domestique Musca domestica L. (Diptera Muscidae). Journal of Applied Biosciences 55: 3953-3961.

8. Corbel V., N'guessan R., Brengues C., Chandre F., Djogbenou L., Martin T., Akogbéto M., Hougard J.M. \& Rowland M. 2007. 
Multiple insecticide resistance mechanisms in Anopheles gambiae and Culex quinquefasciatus from Benin, West Africa. Acta tropica 101: 207-216.

9. David J.-P., Strode C., Vontas J., Nikou D., Vaughan A., Pignatelli P.M. \& Louis C., Hemingway J. \& Ranson H. 2005. The Anopheles gambiae detoxification chip: A highly specific microarray to study metabolic-based insecticide resistance in malaria vectors. Proceedings of the National Academy of Sciences of the United States of America 102: 4080-4084.

10. Fotedar R., Banerjee U., Singh S., Shiriniwa S., Verma A., 1992: The housefly (Musca domestica) as a carrier of pathogenic microorganisms in a hospital environment. J Hosp Infect 20 : 209-215.

11. Garima Z. \& Amla Batra, 2011 : Thevetia peruviana (Pers.) Schum: A plant with enormous therapeutic potential. Journal of Pharmacy Research 2011,4(12),4461-4464.

12. Georghiou G. P., Ariaratnam V., Pasternak M. E., Lin C. S., (1975). Organophosphorus multiresistance in Culex quinquefaciatus in California. J. Econ. Entomol. 1975 Aug;68(4):461-7.

13. Greenberg B, 1971: Flies and disease, vol. I.Ecology,classification and biotic associations. Princeton University Press, New Jersey: 1214.

14. Wanyika H.N., Kareru P.G., Keriko J.M., Gachanja A.N, Kenji G.M. \& Mukiira N.J, 2009: Contact toxicity of some fixed plant oils and stabilized natural pyrethrum extracts against adult maize weevils (Sitophilus zeamais Motschulsky). African Journal of Pharmacy and Pharmacology Vol. 3(2). pp. 066-069.

15. Idrissi H. L. M. \& Hermas J., 2008 : Effet de 1'alimentation en Peganum harmala L. (Zygophyllaceae) sur le tube digestif du criquet pèlerin Schistocerca gregaria Forsk. (Orthoptera, Acrididae). Zool. Baetica, vol. 19: 71-84.

16. Mollah J. U. \& Islam W., 2007: Toxicity of Thevetia peruviana (Pers) Schum. Extract to Adults of Callosobruchus maculatus F. (Coleoptera: Bruchidae). Agric Rural Dev 5(1\&2), 105-109.

17. Kareru P.G., Keriko J.M., Kenji G.M., Thiong’o G.T, Gachanja A. N. \& Mukiira H.N., 2010: Antimicrobial activities of skincare preparations from plant extracts. Afr. J. Trad. CAM (2010) 7 (3): 214 -218 .

18. Kemassi A., Boual Z., Lebbouz I., Daddi Bouhoun M., Saker M.L.,. Ould El Hadj-Khelil A. et Ould El Hadj M.D., 2012: Étude de l'activité biologique des extraits foliaires de Cleome arabica (Capparidaceae). Lebanese Science Journal, vol. 13(2): 81-97. 
19. Kemassi A., Boual Z., Ould El Hadj- K. A., Dadi Bouhoun M. et Ould El Hadj M. D., 2010: Activité biologique de l'extrait d'Euphorbia guyoniana (Boiss. \& Reut.) (Euphorbiaceae) chez le Criquet pèlerin Schistocerca gregaria (Forskål, 1775) (OrthopteraAcrididae). Annales de Sciences et Technologie, Université Kasdi Merbah-Ouargla, vol 2(1):61-70.

20. Kemassi A., Bouziane N., Boual Z., Ould El Hadj M.D., 2014: Activité biologique des huiles essentielles de Peganum harmala L. (Zygophyllaceae) et de Cleome arabica L. (Capparidaceae) sur Schistocerca gregaria (Forskål, 1775). Vol.12 nº 6 (2014): 348-353

21. Magesa S.M., Wilkes T.J., Mnzava A.E.P., Njunwa K.J., Myamba J., Kivuyo M.D.P., Hill N., Lines J.D. \& Curtis C.F., 1991: Trial of pyrethroid impregnated bednets in an area of Tanzania holoendemic for malaria Part 2. Effects on the malaria vector population. Acta Tropica 49: 97-108.

22. Maldonado D. and Centeno N., 2003: Quantifying the potential pathogens transmission of the bowflies (Diptera: Calliphoridae). Mem Inst Oswaldo Cruz 98: 213-216

23. Monzon R., Sanchez R., Tadiaman B., Najos O., Valencia E., De Rueda R., Vatura J., 1991: A comparison of the role of Musca domestica (Linnaeus) and Chrysomya megacephala (Fabricius) as mechanical vectors of helminthic parasites in a typical slum area of Metropolitan Manila. In Southeast Asian Journal Trop Med Public Health 22: 222-228.

24. MS. 2013 : Annuaire des statistiques sanitaires. Archive Ministère de la Santé, pp 87. 116. Müller P., Donnelly M.J. \& Ranson H. 2007. Transcription profiling of a recently colonised pyrethroid resistant Anopheles gambiae strain from Ghana. BMC genomics 8: 36.

25. Müller P., Donnelly M.J. \& Ranson H., 2007: Transcription profiling of a recently colonised pyrethroid resistant Anopheles gambiae strain from Ghana. BMC genomics 8: 36.

26. Neelam R. and Anil K., 2014: Pharmacological Importance of Thevetia peruviana. INTERNATIONAL JOURNAL OF PHARMACEUTICAL AND CHEMICAL SCIENCES ISSN: 22775005

27. Omolara O. Oluwaniyi and Samuel A I., 2007: A Study of the Extractability of Thevetia Glycosides with Alcohol Mixture. Journal ofFood Technology 5 (2): 147 151,2007.

28. Organisation Mondiale de la Sante, 2004: Lutte contre les vecteurs du paludisme. WHO/CDS/WHOPES/2002. 5Rev.1.

29. Organisation Mondiale de la Sante1963 : Méthode à suivre pour déterminer la sensibilité ou la résistance des larves de moustiques aux 
insecticides. In Résistance aux insecticides et lutte contre les vecteurs. Treizième rapport du comité OMS d'experts des insecticides, Genève : OMS, Sér. Rapp. Techn. 265, p. 55-60

30. Ould El Hadj M. D., Tankari D. A., Halouane F. \& Doumandji S., 2006: Toxicité comparée des extraits de trois plantes acridifuges sur les larves du cinquième stade et sur les adultes de Schistocerca gregaria (Forskål, 1775) (Orthoptera-Cyrtacanthacridinae). Sécheresse, vol. 17 (3): 407414.

31. Senegre G., Jilien J. L. \& Gaven B., 1977): Acquisition progressive de la résistance au chlorpyrifos chez les larves de Culex pipiens (L.) dans le midi de la France. Parasitologia 19 (1-2), p. 79-94.

32. Siriwattanarungsee S., Kabkaew L., Olson K., Chailapakul O., Sukontason K., 2008: Efficacy of neem extract against the blowfly and housefly. Parasitol. Res. 103: 535-544.

33. Sukontason K., Buncho M., Klantawa B., Piangjai S., Rongsriyam Y., Sukontason L., 2007: Comparison between Musca domestica and Chrysomya megacephala as carriers of bacteria in northern Thailand. In Southeast Asian Journal Trop Med Public Health 38: 38-44.

34. Sulaiman S., Othman M. and Aziz A., 2000: Isolations of enteric pathogens from synanthropic flies trapped in downtown Kuala Lumpur. J Vector Ecol 25: 90-93.

35. World Health Organization, 1992: Limphatic filariasis: the disease and its control. Fifth report of the expert committee on filariasis. WHO Tech Rep Ser, 821: pp. 1-71.

36. Yadouleton A.W., Padonou G., Asidi A., Moiroux N., Bio-Banganna S., Corbel V., N'guessan R., Gbenou D., Yacoubou I., Gazard K. \& Akogbéto M. C. 2010: Insecticide resistance status in Anopheles gambiae in southern Benin. Malaria Journal 9: 83.

37. Zouiten H., Abbassi K., Atay-Kadiri Z., Mzari M., El Mahi M. \& Essassi E. M., 2006: Insecticidal activity of Solanum sodomaeum (solonaceae) extracts on Schistocerca gregaria (Forskål) larvae. J. Orthop. Res., vol. 15 (2):171-173. 